\title{
Remote-Gating of Schottky Barrier for Transistors and Their Vertical Integration
}

Young Jin Choi, ${ }^{1}$ Seongchan Kim, ${ }^{1}$ Hwi Je Woo, ${ }^{1}$ Young Jae Song, ${ }^{1,2}$ Yoonmyung Lee, ${ }^{3}$ Moon Sung Kang, ${ }^{3, *}$ and Jeong $\mathrm{Ho} \mathrm{Cho}^{4, *}$

${ }^{1}$ SKKU Advanced Institute of Nanotechnology (SAINT), ${ }^{2}$ Department of Nano Engineering, ${ }^{3}$ Department of Electrical and Computer Engineering, Sungkyunkwan University, Suwon 440-746, Republic of Korea.

${ }^{3}$ Department of Chemical and Biomolecular Engineering, Sogang University, Seoul 04107, Korea.

${ }^{4}$ Department of Chemical and Biomolecular Engineering, Yonsei University, Seoul 03722, Korea.

Y. J. Choi and S. Kim contributed equally this work.

E-mail: jhcho94@yonsei.ac.kr and mskang@ssu.ac.kr 


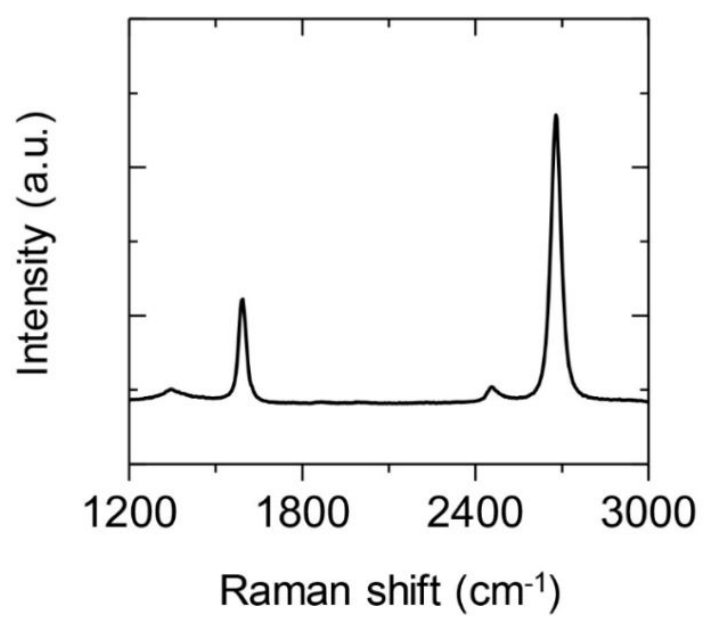

Figure S1. Raman spectrum of CVD-grown monolayer graphene.

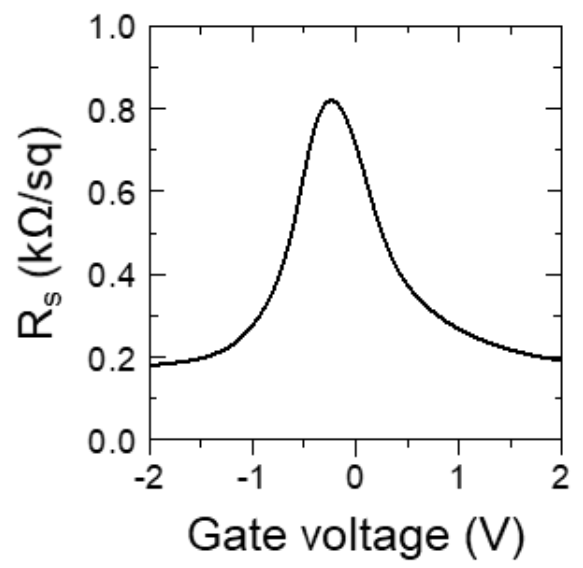

Figure S2. Change in the sheet resistance of a graphene strop induced by $V_{\mathrm{G}}$ applied at the remote gate $(d=$ $50 \mu \mathrm{m})$

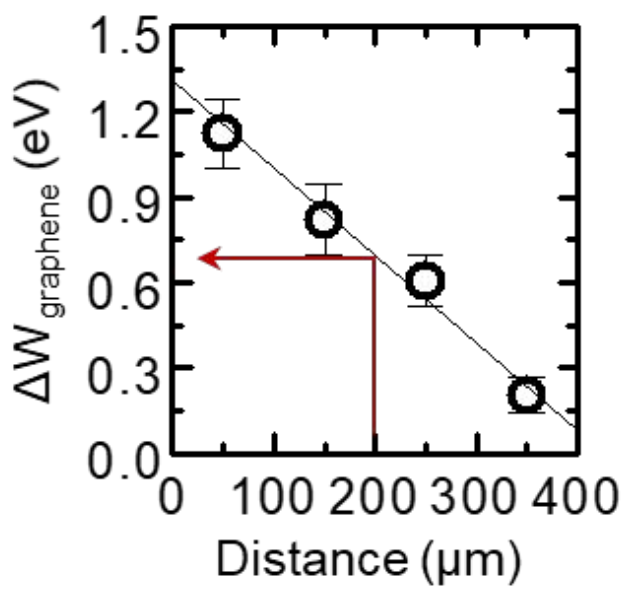

Figure S3. Graphene work function change with remote-gating by ion gel, as a function of distance. 
a

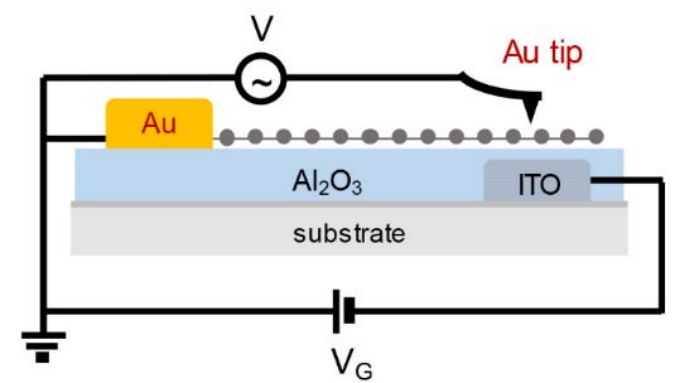

b
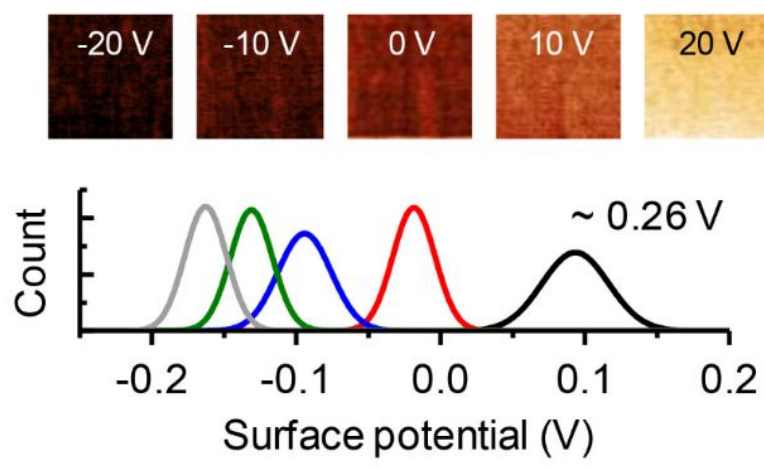

Figure S4. (a) Schematic of measurement setup of KPFM for 110-nm-thick $\mathrm{Al}_{2} \mathrm{O}_{3}$-gated graphene. (b) KPFM images and histogram of surface potential values of graphene at different $V_{\mathrm{G}}$ values $(-20,-10,0,10$, and 20 V).

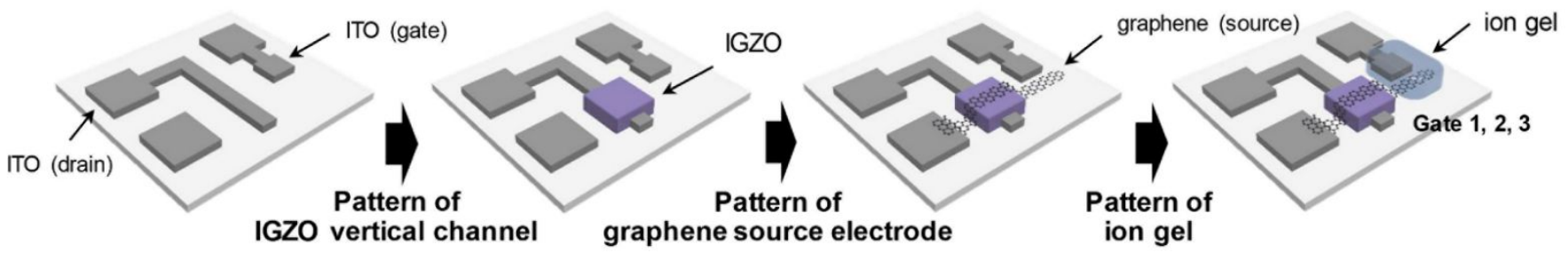

Figure S5. Schematic of the fabrication procedure for IGZO-graphene heterojunction $v$-SBT with ion gel remote-gating. 

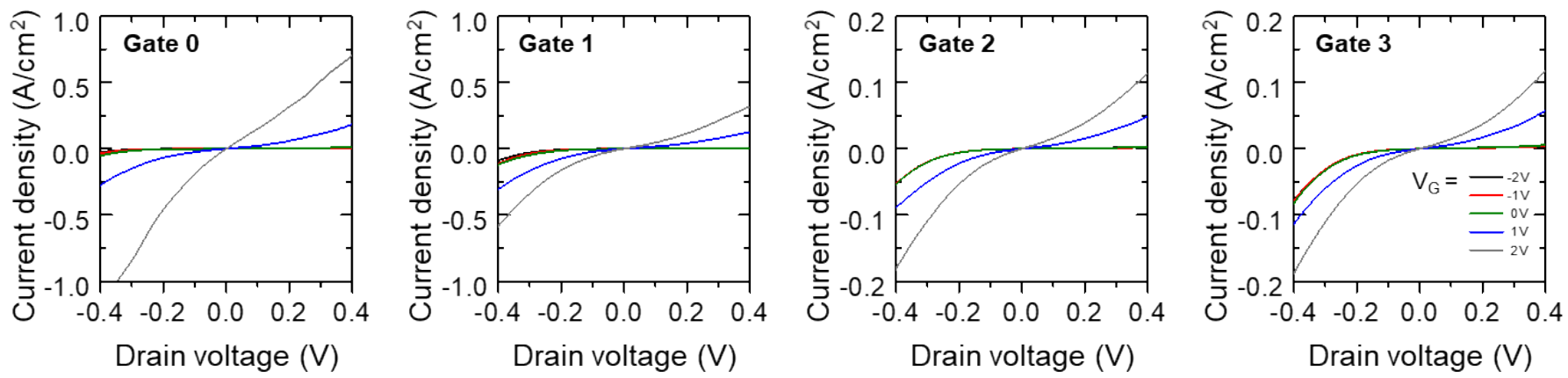

Figure S6. Output characteristics of the $v$-SBT yielding Figure 3d plotted in linear scale
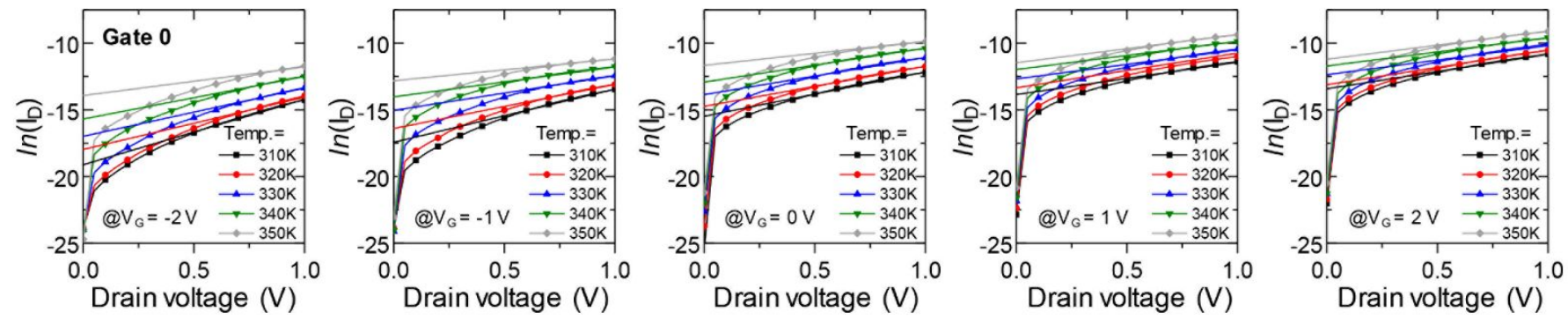

Figure S7. Temperature-dependent output curves of $v$-SBT with Gate 0 at different $V_{\mathrm{G}}$ values $(-2,-1,0,1$, and $2 \mathrm{~V})$.
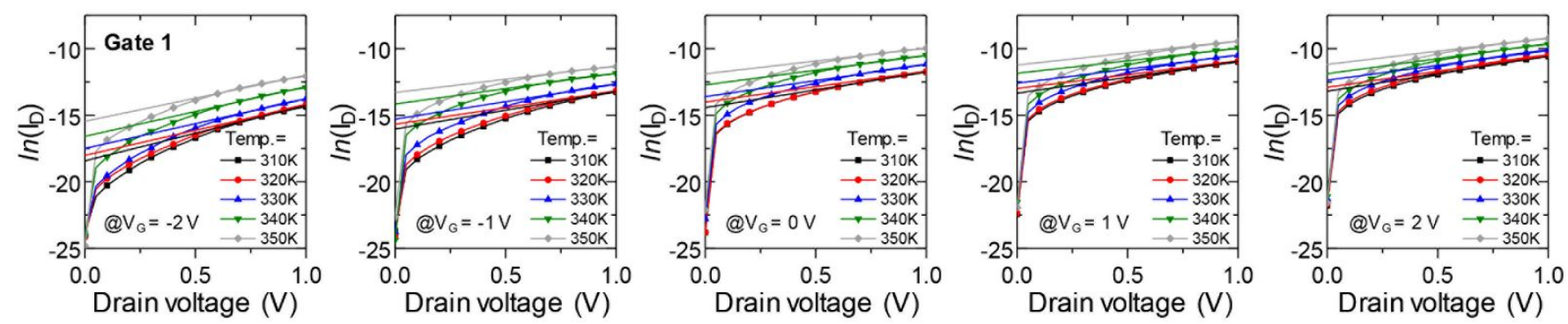

Figure S8. Temperature-dependent output curves of $v$-SBT with Gate 1 at different $V_{\mathrm{G}}$ values $(-2,-1,0,1$, and $2 \mathrm{~V}$ ).
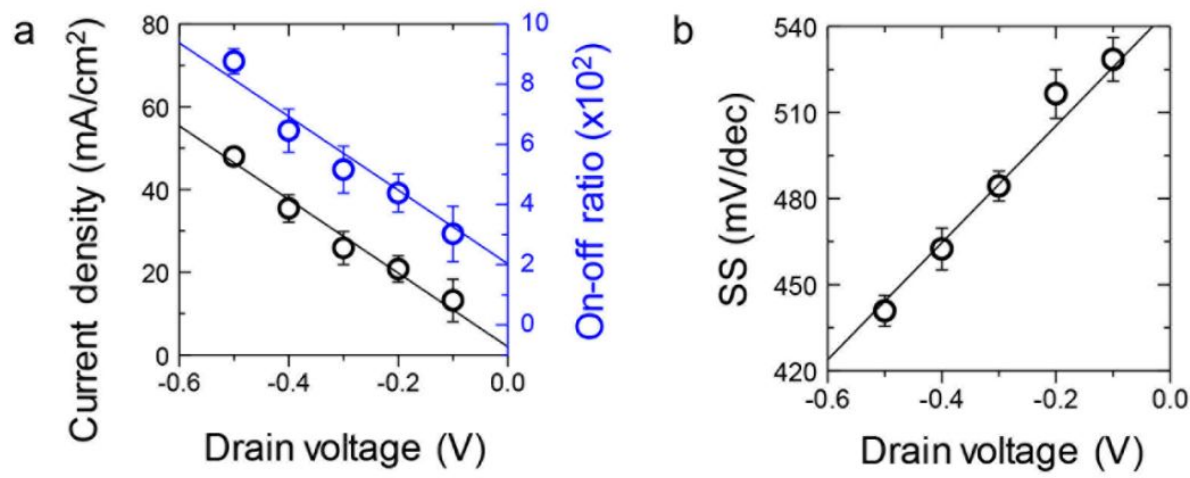

Figure S9. (a) Maximum current density and on-off ratio of pentacene $v$-SBT as a function of $V_{\mathrm{D}}$. (b) Subthreshold swing of pentacene $v$-SBT as a function of $V_{\mathrm{D}}$. 